\title{
A gestão da informação e da comunicação como fatores que influenciam o processo de inovação no contexto colaborativo
}

Thais Zaninelli

\begin{abstract}
Doutora pelo Programa Doutoral em Engenharia Industrial e Gestão da Universidade do Porto, Mestre em Gestão da Informação Universidade do Porto. Bacharel em Biblioteconomia Universidade Estadual de Londrina. Professor Colaborador no Departamento de Ciência da Informação da Universidade Estadual de Londrina
\end{abstract}

Os serviços estão no centro da atividade econômica e a inovação em serviços tem ganhado crescente atenção. Dada à complexidade envolvente no processo de desenvolvimento de novos serviços (DNS), este pode ser realizado através da colaboração de uma rede de empresas. Torna-se, cada vez mais relevante, promover pesquisas globais de serviços que, concomitantemente, desenham uma pesquisa interdisciplinar e cross-funcional de acadêmicos e executivos. Esta pesquisa objetivou entender o complexo processo de DNS e como ele acontece no contexto das redes colaborativas, contribuindo para entender como as redes colaborativas podem potencializar o processo de inovação em serviços. Esta pesquisa teve início com um estudo exploratório, envolvendo quatro complexos projetos de serviços, com o objetivo de caracterizar as principais fases do processo e analisar quando as redes são formadas. Após isso, um estudo qualitativo foi realizado para se ter uma compreensão do papel que as redes têm no desempenho do DNS. Este estudo identificou desejáveis fatores de desempenho do processo de inovação, com o foco na importância da gestão da informação e da comunicação para o sucesso do serviço. O estudo mostrou que alguns fatores são transversais a todo o processo, entretanto, a relevância dos fatores se altera ao longo das fases.

Palavras-chave: Inovação; Desenvolvimento de Novos Serviços; Redes colaborativas; Fatores de desempenho; Gestão da informação; Gestão da comunicação. 


\section{Information and communication management as factors that influence the innovation process in the collaborative context}

Services are at the center of the economic activity and service innovation has gained increased attention. Given the complexity involved on new service development (NSD), it can be carried out by the collaboration of a network of companies. It becomes increasingly relevant to promote global service research agendas that concomitantly draw on the interdisciplinary and crossfunctional perspective of academics and executives. This research aimed to understand the complex NSD process and how this process happens in the Collaborative context, contributing to understand how CNs can potentiate NSD. The research started with an exploratory study involving four service projects with the objective of charactering the main stages of the process and to analyze when CNs were formed. After this, a qualitative study was undertaken to get an in-depth understanding of CNs role in the NSD performance. This study identified desirable performance factors of NSD in the CNs context in large projects, analyzing how the factors evolve and how they influence the stages along the NSD process, with focus on importance of information and communication management to the process success. The study showed that some performance factors cross-cut all of NSD process, however, the relevance of these factors change along these stages.

Keywords: Innovation; New Service Development; Collaborative networks; Performance factors; Information management; Communication anagement.

Recebido em 11.09.2012 Aceito em 21.05.2013

\section{Introdução}

Durante as duas últimas décadas, a desregulamentação e a globalização dos mercados tornaram a competição entre as empresas de serviços extremamente feroz. Essas tendências em torno da inovação nos 
serviços ocupam um lugar no coração da competitividade das empresas, com uma constante adaptação em um ambiente turbulento, que requer um fluxo contínuo de novas ofertas (STEVENS; DIMITRIADIS, 2005).

Inovação em serviços está agora no centro da atividade econômica (MANSURY; LOVE, 2008) e o processo de Desenvolvimento de Novos Serviços (DNS) ganhou maior atenção (STEVENS; DIMITRIADIS, 2005). Nesse contexto, torna-se relevante promover a pesquisa na área de serviços, que, concomitantemente, desenha uma perspectiva interdisciplinar para acadêmicos e executivos de negócios (OSTROM et al., 2010).

Um complexo DNS, tal como os centros comerciais, envolve várias fases, que vai desde a ideia de um novo Centro Comercial até o seu lançamento no mercado (EDVARDSSON et al., 2000). Devido à complexidade envolvente em todo esse processo, torna-se difícil uma única empresa obter "in house" todas as competências e processos necessários para desenvolver o novo serviço, de forma otimizada e completa, tornando-se cada vez mais comum e necessário 0 estabelecimento de relacionamentos com empresas externas, através das redes colaborativas, para potencializar o processo de DNS (VALKEAPAA et al., 2006).

A colaboração entre empresas tem sido pouco estudada e explorada no contexto da framework das redes de colaboração (CAMARINHAMATOS; AFSARMANESH, 2008), principalmente o papel da gestão da informação e da comunicação - como fatores de qualidade da rede - entre essas empresas participantes na rede colaborativa para o DNS.

Grande parte da literatura aborda o DNS e as redes colaborativas de forma separada e poucos estudos avaliam o processo de DNS no contexto das redes. Este artigo apresenta resultados de um estudo de caso exploratório e um estudo qualitativo dos fatores de qualidade das redes colaborativas que influenciam o desempenho do processo de DNS ao longo das suas diferentes fases, no contexto das redes.

Dessa forma, o estudo contribui para um melhor entendimento de como as redes colaborativas podem potencializar o processo e influenciar o desempenho do DNS e, por consequência, o sucesso do serviço final, e, também, como que os fatores de qualidade das redes evoluem ao longo das diferentes fases do processo. Os resultados contribuem para: (1) integrar pesquisa sobre DNS e sobre Redes colaborativas em um mesmo contexto; (2) cooperar com novos desafios em projetos complexos de DNS, tal como é um Centro Comercial; e (3) gerar recomendações para gestão da rede, no que tange a gestão da informação e comunicação entre as empresas que trabalham juntas na rede, quando do complexo processo de DNS.

\section{Revisão de literatura}

\subsection{Inovação em serviços e processo de Desenvolvimento de Novos Serviços}


Desenvolvimento de Novos Serviços (DNS) pode ser definido, na literatura, como "um conjunto de atividades, ações e tarefas que movem um projeto desde a fase da geração da ideia de um novo serviço até seu lançamento" (ALAM; PERRY, 2002).

Muitas abordagens para o processo de DNS ainda se originam do campo de Desenvolvimento de Novos Produtos (DNP) (SCHEUING; JOHNSON, 1993; AKAMAVI, 2005), entretanto, o processo requer adaptações no contexto do serviço. Vários autores defendem que o DNS deve seguir um processo estruturado, incluindo um conjunto de atividades, que são feitas de forma sequencial ou em paralelo (TAX; STUART,1997).

No modelo proposto por Edvardsson et al. (2000), o processo de DNS é composto por quatro fases genéricas: (1) Geração da ideia do novo serviço; (2) Estratégia de desenvolvimento do novo serviço; (3) Desenho do serviço; e (4) Desenvolvimento e implementação do novo serviço.

Nesse contexto, um número notável de pesquisadores tem buscado identificar alguns fatores que são cruciais para o desempenho do processo de DNS. Tais fatores podem ser divididos em: (1) internos; e (2) externos (OJANEN et al., 2008).

Fatores internos estão associados a forças e fraquezas da empresa, tais como: inovação, competência central, desenho do processo de DNS (FROEHLE et al., 2000; VARGO; LUSCH, 2004). Os fatores externos, por sua vez, estão relacionados aos benefícios do novo serviço e se suas características atendem às necessidades do mercado. Esses fatores incluem, por exemplo, o fit entre as necessidades dos clientes e os benefícios da oferta do novo serviço, bem como o fit entre o novo serviço e o portfólio dos que já existem (VARGO; LUSCH, 2004).

Devido à complexidade envolvente no DNS, o processo é considerado, por vezes, desafiador, dadas as relações intra e extra organizacional, que são necessárias gerir (ALBRECHT, 2000). Estudos nas últimas duas décadas têm revelado que muitos fatores de sucesso ou falência do DNS não têm diferenças significativas em relação aos fatores que influenciam o processo de DNP, tais como: características do produto, orientação ao mercado (JAW et al., 2010), o foco estratégico na inovação (EDVARDSSON et al., 1995; JOHNE; STOREY, 1998), alocação de recursos apropriados (EDGETT, 1994; JONG; VERMEULEN, 2003), gestão da informação e comunicação (ZANINELLI; PATRÍCIO; FALCÃO e CUNHA, 2010), gestão de suporte (MARTIN JR; HORNE, 1995). Porém, as típicas características do serviço: intangibilidade, heterogeneidade, inseparabilidade e perecibilidade podem ter uma influência significante na ênfase desses fatores de desempenho do DNS (OJANEN et al., 2008).

Pesquisas existentes têm estudado a importância desses fatores de desempenho para o sucesso do processo de DNS. Entretanto, prévios estudos não têm analisado esses fatores quando o processo acontece no contexto das redes colaborativas ou, ainda, como esses fatores evoluem ao longo das diferentes fases do processo de DNS. 


\subsection{Redes colaborativas}

Uma rede colaborativa é criada por muitas entidades (organizações e pessoas), que são, em grande parte, autônomas, estão geograficamente distribuídas e que são heterogêneas em termos de: (1) ambientes operacionais, (2) cultura, (3) capital social e (4) objetivos (CAMARINHAMATOS; AFSARMANESH, 2008). Essas entidades colaboram para melhor atingir objetivos comuns e objetivos compatíveis (LEWIS, 1992; ROSENFELD, 1997).

As redes colaborativas podem ser formadas por organizações através de parcerias entre empresas, equipes virtuais ou acordos estratégicos, em que empresas deliberadamente decidem iniciar um projeto comum, embora permaneçam independentes depois do contrato (LIPNACK; STAMPS, 1994).

Prévias pesquisas indicam que apenas uma em cada cinco organizações tem orientações pré-estabelecidas para manter o sucesso do relacionamento em rede (AUSTIN; 2000). A gestão formal desse processo é ainda escassa e raramente eficaz. Esses estudos mostram que a gestão da rede requer um relacionamento com troca de valores, ideias e confiança, o que se traduz na construção de um forte compromisso entre todas as partes envolvidas na rede.

Portanto, é desejável uma gestão estratégica da rede, bem como uma forte e estruturada gestão da informação e da comunicação, que seja transversal a todos os participantes da rede. Pois, esses fatores desempenham um papel essencial para o sucesso da relação a longo prazo, capaz de permitir que os parceiros obtenham um desempenho superior e uma vantagem competitiva sustentável e garantam o desempenho do processo e, por conseguinte, o sucesso do novo serviço quando lançado ao mercado (AUSTIN, 2000).

Nesse contexto, com base em extensa literatura, a qualidade da rede colaborativa é avaliada dentro de duas dimensões: (1a) Fatores de qualidade das empresas parceiras na rede, tais como: (a) experiência prévia em parcerias (ANAND; KHANNA, 2004; EMULT; KATHAWALA, 2001), (b) flexibilidade (LÉON; AMATO NETO, 2001); e (2a) Fatores de qualidade do relacionamento entre as empresas, tais como: (a) gestão dedicada da rede (AUSTIN, 2001), (b) gestão da informação e da comunicação (ZANINELLI; PATRÍCIO; FALCÃO e CUNHA, 2010; MAGLIO, 2010), e (c) confiança (AUSTIN, 2000; JÚNIOR; RIBEIRO, 2001).

As redes colaborativas necessitam de futuras pesquisas, dado 0 papel crucial que elas têm no desempenho do processo de DNS e no sucesso do serviço final. Extensa literatura aborda o processo de DNS e as redes colaborativas de forma separada e ambas as áreas não estão bem conectadas. Portanto, futuras pesquisas necessitam identificar como as redes podem promover o processo de DNS ao longo das suas diferentes fases. 


\section{Procedimentos metodológicos: etapas percorridas, métodos e instrumentos adotados}

A gestão da rede colaborativa, no contexto do processo de DNS, é uma área de pesquisa nova - e, por isso, pouco explorada - além de complexa. O presente estudo adotou a pesquisa qualitativa (SAMPIERI et al., 2006), uma vez que é considerada a mais adequada para fornecer em profundidade o entendimento desse novo fenômeno. A primeira fase da pesquisa envolveu um estudo exploratório em quatro projetos de Centros Comerciais de grande dimensão.

Esses projetos foram selecionados para cobrir quatro grandes fases do processo de DNS: (1) Fase da geração de ideia de um novo serviço e fase do desenvolvimento do conceito do serviço (fases iniciais); (2) Fase da construção; (3) Fase do lançamento; e (4) Fase da operação. Esses quatro Centros Comercias representaram projetos complexos de serviços, envolvendo redes de empresas parceiras; cada projeto estava em diferentes fases do processo de desenvolvimento.

O objetivo do estudo exploratório (SAMPIERI et al., 2006), desenvolvido com base no método estudo de casos múltiplos (YIN, 2009), foi identificar e caracterizar as fases do processo de DNS e analisar quando e como as redes colaborativas são formadas ao longo desse processo.

Nessa fase da pesquisa, ou seja, a exploratória, a coleta de dados envolveu entrevistas com os gestores da empresa desenvolvedora. A coleta de dados por meio de entrevistas foi completada através da observação do processo de seleção das empresas parceiras e análise de documentos, tais como: relatório técnico, contrato de parcerias e projetos conceituais. Nessa fase, foram identificados e definidos os gestores que seriam entrevistados na segunda fase da pesquisa, isto é, o estudo qualitativo. O principal critério de seleção desses gestores foi a sua participação direta no processo de DNS.

A segunda fase da pesquisa objetivou identificar os fatores de qualidade da rede e entender como esses fatores influenciam 0 desempenho do processo. O estudo qualitativo envolveu 39 entrevistas em profundidade com gestores das empresas parceiras e da desenvolvedora, que foram previamente definidas no estudo exploratório. Seguindo com o método qualitativo Grounded Theory (CHARMAZ, 2006), as entrevistas foram gravadas, literalmente transcritas e analisadas com 0 suporte do programa de análise de conteúdo qualitativo, o Nvivo. As informações foram coletadas em um processo interativo, com base nos objetivos da pesquisa: (1) Fases do processo de DNS; (2) Redes colaborativas no processo de NSD; e (3) Fatores de qualidade das redes.

A análise das informações das duas fases permitiu um entendimento em profundidade do papel da rede no processo de DNS por ambas as perspectivas: empresas parceiras e desenvolvedora, bem como identificou e descreveu um rico conjunto de fatores que influenciam o desempenho do processo de DNS ao longo das diferentes fases. 


\section{Resultados}

\subsection{Onvolvimento de redes ao longo das diferentes fases do processo de DNS}

O estudo exploratório de casos múltiplos permitiu uma melhor compreensão de quais são as atividades e as fases envolventes no processo de DNS, bem como o entendimento do papel das redes ao longo das diferentes fases do processo. O estudo mostrou que o processo de DNS nos quatro projetos complexos segue um processo cíclico. Isso significa que o processo de desenvolvimento de novos serviços não termina, pois está em constante evolução.

Com base nos resultados, cinco principais fases foram identificadas no ciclo do processo: (1) geração da ideia; (2) desenvolvimento conceitual do projeto - as fases 1 e 2 são definidas como fases iniciais; (3) construção; (4) lançamento; e (5) operação. Porém, os resultados do estudo revelaram que, depois que o serviço é lançado ao público, a fase da operação tem início, portanto, o processo não termina. A figura 1 , a seguir, representa o processo e suas respectivas fases.

Figura 1 - O processo de DNS

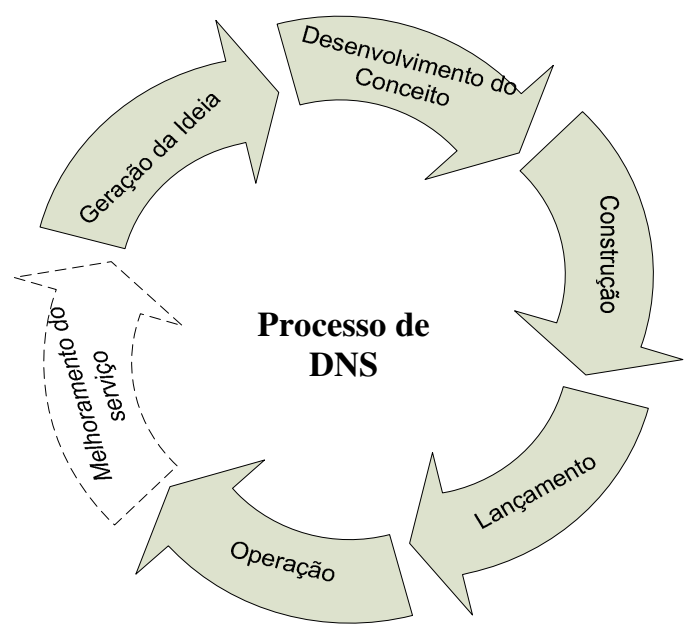

Fonte: ZANINELLI (2011, p. 88).

A fase da geração da ideia é o ponto de partida do processo, seguida da fase de desenvolvimento do conceito. O estudo mostrou que ambas as fases têm características específicas e as informações geradas nas referidas fases exercem influência direta nas fases seguintes do processo. Tais fases iniciais são desenvolvidas através de diferentes departamentos da empresa desenvolvedora e as atividades por seus respectivos colaboradores. Dessa forma, o estabelecimento de redes colaborativas, nessas fases iniciais do DNS, é praticamente inexistente.

A equipe que desenvolve essas fases é composta por expertises e, de acordo com Edvardsson et al. (2000), é de extrema importância uma 
equipe ser especializada e multidisciplinar, quando dos complexos projetos de DNS. Os resultados revelam que essas fases são o início do processo e geram informações que servirão de base para o desenvolvimento das seguintes. Portanto, as primeiras fases influenciam o desenvolvimento das fases seguintes do processo e, por consequência, têm um significante impacto no sucesso do serviço quando do seu lançamento.

O desenvolvimento do conceito é a fase de transformar a ideia do novo serviço - um conceito intangível - em um conceito tangível, com especificações concretas da oferta do novo serviço, descrevendo a proposta de valor que a empresa quer oferecer para futuros clientes.

As atividades envolventes na fase de desenvolvimento do conceito, ou seja, a definição da oferta central do serviço, envolvem: estudo de mercado, estudo das características dos potenciais consumidores, análise das características da região onde o serviço será ofertado, análise dos potenciais concorrentes, definição da localização geográfica e desenho conceitual do novo serviço.

As atividades são desenvolvidas "in-house", sem intervenções de parceiros externos, uma vez que o desenvolvimento do conceito do novo serviço envolve o core business da empresa desenvolvedora, delegando outras atividades dessa fase às empresas parceiras, através do outsourcing estratégico. As empresas parceiras são contratadas no contexto das redes colaborativas para transformar o desenho do conceito em projetos executivos detalhados. Esses projetos variam de iluminação até projetos de arquitetura, dependendo do tipo e das características do serviço.

O relacionamento colaborativo, desde as fases iniciais do processo, fornece uma redução no tempo e no custo do processo. Por um lado, as parceiras são especialistas em várias atividades que são necessárias para desenvolver o conceito do serviço de forma completa. Por outro lado, o conceito central do serviço é desenvolvido internamente e criado pela empresa desenvolvedora para preservar o conhecimento estratégico e core business, que são crucias para manter a vantagem competitiva da empresa.

A próxima fase envolve a construção do espaço físico onde o serviço será ofertado. Essa fase define importantes atributos físicos para a percepção do cliente relativamente à qualidade do serviço. A construção do espaço é feita com base nas informações geradas nas fases anteriores do processo, nomeadamente: geração da ideia e desenvolvimento do conceito. Portanto, nessa fase do processo, tudo o que foi definido nas fases iniciais tornam-se projetos executáveis. Tal transição envolve a consolidação do projeto conceitual, ou seja, as soluções são desenvolvidas para colocar em prática a construção do espaço físico onde o serviço vai ser ofertado.

As principais atividades que compõem essa fase são: desenvolvimento de projetos executivos, desde arquitetura até projetos de ar condicionado (como já mencionado, estes projetos variam de acordo com o tipo de serviço que será ofertado), atividade de marketing do novo 
serviço e comercialização. A gestão das evidências físicas, tais como equipamentos, sinalização, cheiro, decoração, temperatura, etc., influencia positivamente a experiência do cliente ao longo do seu encontro com o serviço.

A fase seguinte da construção envolve o lançamento do novo serviço, que acontece em dois momentos. O primeiro está relacionado ao lançamento do serviço para as empresas parceiras que vão oferecer serviços suplementares, ou seja, aqueles serviços que adicionam valor à oferta do serviço central, como, por exemplo, as lojas de varejo. Esses produtos e serviços são denominados de suplementares e, muitas vezes, são mais importantes que o serviço central, uma vez que podem assegurar a diferenciação da oferta central, especialmente em regiões onde o serviço central já seja oferecido por empresas concorrentes (NORMANN, 1993). O segundo momento está associado ao lançamento do serviço ao consumidor final.

Nessa fase, todos os componentes do serviço, tais como trabalhadores, estruturas funcionais, serviços suplementares, espaços físicos, sistemas técnicos e processos, devem estar prontos. Todos esses componentes devem formar um conjunto harmônico e trabalharem juntos para garantir o lançamento do serviço para o público e a operação iniciar com sucesso.

$\mathrm{Na}$ fase do lançamento, uma nova rede colaborativa é formada e novos relacionamentos são estabelecidos com empresas parceiras, que são responsáveis pela gestão e manutenção do novo serviço, depois de lançado ao público. Essa gestão envolve atividades, como: limpeza, equipamento técnico de manutenção (ar condicionado, elevadores, câmeras de segurança), reciclagem, dentre outras atividades que variam de acordo com o tipo de serviço que a empresa vai oferecer. Tais atividades são cruciais para a qualidade do serviço e para a primeira impressão do serviço pelo cliente. Nessa fase, inicia-se a contratação de colaboradores que irão fazer parte do serviço em operação, que vão desenvolver diferentes funções que garantam a qualidade do serviço para o cliente.

A operação do serviço, ou seja, o momento em que ele é ofertado para os clientes finais, é considerada uma fase do processo, uma vez que o DNS é cíclico (JOHNSON et al., 2000). Alguns pesquisadores consideram esse momento como "the moment of truth" (EDVARDSSON et al., 2000; GRONROOS, 2000; FROEHLE; ROTH, 2007), ou seja, o momento da verdade, momento em que os clientes vão consumir o serviço e avaliar sua qualidade.

Os resultados dessa fase são influenciados pela participação do cliente e algumas características, tais como: segurança, conforto, limpeza, etc., que se tornam requisitos essenciais para a satisfação do cliente. Nessa fase, alguns fatores, que até o momento não eram relevantes, são mais evidentes, tais como: tendências, cultura, características locais dos clientes e competidores locais. Os referidos fatores e a influência deles no 
desempenho do serviço não podem ser totalmente previstos nas fases iniciais do processo.

O objetivo dessa fase é coordenar as atividades demandadas e criar valor para o cliente. Portanto, as principais atividades relacionadas à gestão de um complexo serviço do tipo Centro Comercial, quando da operação, envolvem atividades de entrega e consumo para os clientes, tais como: gestão de operações técnicas, gestão das empresas parceiras, gestão dos lojistas e atividades de marketing. Nesta mesma linha, o resultado do estudo mostra que a base para o sucesso de todas essas vertentes da gestão é a intensa comunicação e um estruturado fluxo da informação que seja transversal a todas as partes envolvidas no processo.

\subsection{Fatores de qualidade das Redes Colaborativas ao longo do Processo de DNS}

O estudo qualitativo permitiu identificar e melhor entender quais são os fatores que qualificam uma rede colaborativa. Os resultados mostram que os fatores de qualidade das redes colaborativas são classificados dentro de duas dimensões: (1 $\left.{ }^{a}\right)$ Características das empresas parceiras e (2a) Características da relação entre as empresas.

\subsubsection{Qualidade da rede colaborativa: características das empresas parceiras}

Características das empresas estão relacionadas aos atributos que as mesmas devem satisfazer para participarem de uma rede colaborativa em projetos complexos de DNS. Experiência prévia em parceria, Knowhow, cumprimento das regras e normas do contrato, boa reputação no mercado, flexibilidade e foco na sustentabilidade foram apontados como características desejáveis que as empresas parceiras devem ter especificamente e que contribuem para o bom desempenho do processo e para o sucesso do serviço, quando ele é lançado ao público. Essas e outras dimensões das características das empresas são apresentadas na Tabela 1. 
Tabela 1 - Características das empresas

\begin{tabular}{lc}
\hline \multicolumn{1}{c}{$\begin{array}{c}\text { REDE COLABORATIVA } \\
\text { Características das empresas }\end{array}$} & $\begin{array}{c}\text { Geral } \\
(\mathbf{n = 3 9 )}\end{array}$ \\
\hline Experiência em Parcerias & $74 \%$ \\
Know-how & $64 \%$ \\
Cumprimento de regras e normas do & $64 \%$ \\
contrato & \\
Reputação no mercado & $59 \%$ \\
Flexibilidade & $51 \%$ \\
Foco na sustentabilidade & $49 \%$ \\
Qualidade do serviço & $46 \%$ \\
Pró-atividade & $44 \%$ \\
Foco no cliente & $38 \%$ \\
\hline
\end{tabular}

Fonte: Dados da pesquisa.

Experiência em parcerias está relacionada à prévia experiência das empresas em trabalharem juntas através de redes ou outro tipo de colaboração entre empresas para desenvolver produtos e serviços. Ter experiência em prévios projetos fortalece a relação em rede e capacita a resolução de possíveis conflitos no contexto das atividades envolventes no processo, devido a experiências passadas que podem servir de exemplos para o desenvolvimento de novos projetos.

Empresas com prévias experiências em parcerias têm uma forte capacidade de gerir fontes internas e fontes externas, tornando fácil a resolução de possíveis conflitos que podem vir a ocorrer durante a relação em rede (ANAND; KHANNA, 2004).

Know-How também foi apontado como uma importante característica que as empresas parceiras devem ter para ajudar a potenciar o processo de DNS. Do ponto de vista dos entrevistados, Knowhow envolve o conhecimento das empresas, onde esses entrevistados trabalham, o conhecimento que eles têm de uma área específica e, também, o quanto acreditam que sua empresa é melhor que as outras nessa atividade. Para os entrevistados, esse conhecimento é a razão pela qual sua empresa é selecionada para trabalhar na rede no processo de grande dimensão, tal como o DNS. De acordo com o gestor de uma loja de varejo, que trabalha na fase de lançamento do serviço:

[...] o primeiro shopping foi um sucesso, mas nos dia de hoje em Portugal existem muitos shoppings. $O$ que acontece é que a empresa desenvolvedora tem sucesso pela sua experiência e Know-how no desenvolvimento de shopping. Onde eles operam, eles têm sucesso.

Portanto, as empresas que possuem Know-how em uma determinada atividade são vistas como empresas de valor em processos 
de DNS, uma vez que know-how tem um forte impacto no desempenho do DNS. Dessa forma, as empresas com conhecimentos específicos ajudam a otimizar o processo e, por consequência, o sucesso final do serviço.

Em projetos de grande dimensão, tal como o DNS, em adição ao Know-how e à experiência em parcerias, o cumprimento das regras e normas do contrato também foi identificado como uma característica relevante para o desempenho do DNS. Tal característica é extremamente importante para trabalhar no contexto das redes, uma vez que existem muitas empresas trabalhando ao mesmo tempo em um mesmo projeto, desta forma, respeitar as normas e regras estabelecidas nos contratos torna-se mais crítico do que quando a empresa atua sozinha no mercado.

Esse fator assume um papel essencial para desenvolver uma coordenação estruturada do processo e ajuda atingir algumas metas do projeto, como, por exemplo, a data de entrega. Desta forma, ajuda o projeto ser desenvolvido em harmonia e no tempo planejado. De acordo com o gestor de uma loja de varejo que iniciou suas atividades na fase de lançamento:

[...] Uma forte e ativa gestão do mall é que faz a empresa desenvolvedora ter sucesso em seus centros comerciais. $E$ para mim, essa gestão que dita o sucesso de todas as coisas. Os gestores não se acomodam, eles não deixam o espaço se degradar, ou seja, eles seguem com todo o rigor as regras e contratos, são exemplos para nós parceiros.

Empresa de grande dimensão tem processos pré-estabelecidos que envolvem normas e padrões devido à complexidade de suas atividades e sua imagem no mercado. Desta forma, a reputação da Marca está relacionada à reputação da empresa no mercado onde ela está inserida e é uma característica muito importante para o desempenho do processo de DNS, especialmente quando esse serviço é lançado para o consumidor final.

Empresas com boa reputação no mercado têm maior possibilidade para estabelecer parcerias, uma vez que essas empresas já são conhecidas no mercado por ambos, consumidores e competidores. Por outro lado, para as empresas menos conhecidas, esse fator é crucial, uma vez que, ao associarem sua imagem a uma grande empresa, acabam por se tornarem mais conhecidas.

Em adição a todos esses fatores de qualidade já mencionados, as empresas parceiras devem ser flexíveis devido à complexidade envolvente no processo de DNS e a rápida mudança das tendências do mercado. As empresas necessitam ter flexibilidade para adaptar seus próprios processos para atenderem rapidamente estas possíveis mudanças.

Desta forma, a flexibilidade está relacionada com a capacidade das empresas parceiras adaptarem seus processos para atender ambos: tendências do mercado e necessidades dos clientes, bem como possíveis modificações do processo de DNS. Nesta mesma linha, uma vez que 
projetos complexos são desenvolvidos dentro de um longo tempo, devido às várias atividades e tarefas envolventes no projeto, essas atividades são muito complexas, tendo em vista a grande dimensão de um serviço do tipo Centro Comercial. Portanto, esses projetos tendem a gerar mais resíduos, que podem causar um grande impacto ambiental, do que projetos com menor dimensão.

Nesse contexto, a sustentabilidade é, portanto, outro fator importante mencionado pelos entrevistados, e está relacionado a processos autossustentáveis e amigos do meio ambiente. Esse fator cruza as diferentes fases do processo de DNS e todas as empresas que trabalham na rede necessitam desenvolver seus processos, tendo em conta a preservação do meio ambiente e, assim, o novo serviço torna-se autossustentável desde o início. Destarte, a capacidade de inovação também é considerada uma característica essencial para a empresa que trabalha na rede no contexto de DNS, uma vez que as necessidades dos clientes, tendências de mercado e tecnologia estão constantemente em mudança e isso requer constante investimento por parte das empresas.

Empresas que inovam são aquelas capazes de desenvolver novas ideias constantemente, com o objetivo de melhorar o processo de desenvolvimento de um novo serviço, seja de forma radical ou incremental, e, dessa forma, conseguem obter uma vantagem acima dos competidores.

Nesse contexto, outra importante característica das empresas parceiras apontada nas entrevistas e que está associada à inovação é a qualidade da oferta do serviço e 0 foco no cliente. Ambas as características se completam, uma vez que a qualidade dos serviços depende da habilidade em ter seu foco no cliente e oferecer serviços que satisfaçam suas necessidades. De acordo com um gestor de uma empresa de serviço da fase da operação:

[...] nós temos condições de oferecer um serviço completo no mall, porque nós conhecemos o mercado e nós executamos e fornecemos nossos serviços com foco no cliente. Nós temos know-how e conhecimento para servir de forma excelente em qualquer lugar no Brasil e isso se traduz num serviço de alta qualidade.

Dessa forma, a qualidade da oferta do serviço e o foco nas necessidades dos clientes por todas as parceiras exercem um impacto direto no sucesso do processo e no serviço final.

Com base no que foi mencionado até o momento, em um complexo projeto de DNS, uma rede colaborativa é composta por várias empresas parceiras, que são responsáveis por desenvolver várias atividades. Porém, devido ao grande número de parceiras que trabalham ao mesmo tempo, problemas e conflitos tornam-se inevitáveis. Portanto, essas empresas devem ser capazes de proporem melhoramentos e soluções rapidamente, com o objetivo desses conflitos não influenciarem, de forma negativa, 0 desempenho do processo. Por conseguinte, as empresas, além de serem 
pró-ativas durante todas as fases do processo, devem ter uma intensa e clara comunicação que seja transversal a todos os parceiros no contexto da rede e a gestão da informação dos projetos nos quais elas estão inseridas deve ser estruturada, para que no meio do processo de desenvolvimento nada seja perdido ou mal interpretado, causando um impacto negativo no desempenho do processo e na oferta final do novo serviço.

Desta forma, pró-atividade é um fator crucial e exerce influência na escolha entre uma empresa e outra para fazer parte da rede em processos de novos serviços. Pró-atividade envolve a capacidade das empresas proporem soluções e terem iniciativas em indicá-las para resolver problemas inesperados. Empresas pró-ativas são ágeis e têm rápida capacidade de propor soluções, portanto, são as mais indicadas para trabalharem em processos complexos, uma vez que, como já mencionado, há várias mudanças ao longo das fases e essas mudanças devem ser resolvidas de modo criativo, inovador e rápido.

\subsubsection{Qualidade da rede colaborativa: características do relacionamento}

A característica da relação em rede envolve atributos que classificam o bom relacionamento entre as empresas que trabalham juntas em uma rede no contexto de projetos complexos de DNS. A Tabela 2 mostra as características da relação em rede, identificadas através das entrevistas, que envolvem o aprendizado mútuo, confiança, melhoramento da imagem da empresa, comunicação e partilha de visão.

Tabela 2 - Característica do relacionamento

\begin{tabular}{lc}
\hline \multicolumn{1}{c}{ REDE COLABORATIVA } & Geral (n=39) \\
\hline Aprendizado Mútuo & $69 \%$ \\
Confiança & $62 \%$ \\
Melhoramento da Imagem da empresa & $56 \%$ \\
Gestão da Informação e da Comunicação & $54 \%$ \\
Visão partilhada & $49 \%$ \\
\hline
\end{tabular}

Fonte: Dados da pesquisa.

Uma vez que uma rede colaborativa é um conjunto de organizações independentes que colaboram entre si, através da oferta de produtos e serviços, com o objetivo de atingir objetivos comuns (LEWIS, 1992; ROSENFELD, 1997; CAMARINHA-MATOS; AFSARMANESH, 2008), o aprendizado mútuo acaba por ser um processo natural na colaboração entre as empresas numa rede, uma vez que a troca de informação e o conhecimento entre as empresas parceiras podem influenciar 0 desempenho do processo. 
Aprendizado mútuo envolve a capacidade que as empresas parceiras têm de adquirir novos conhecimentos devido à estreita relação com outras empresas e aplicar tais conhecimentos em sua rotina diária.

Como já referido, um complexo processo de DNS, tal como o desenvolvimento de um Centro Comercial, envolve várias organizações no contexto das redes, com diferentes objetivos e processos ao longo das diferentes fases. Portanto, para além do aprendizado mútuo, a confiança entre todas as empresas parceiras torna-se um fator relevante para um bom relacionamento em rede.

De acordo com os resultados, estabelecer e manter a confiança em uma rede colaborativa é um aspecto desafiador, devido ao número de empresas envolvidas e devido ao fato de cada empresa ter perspectivas e visões diferenciadas de um mesmo problema. Esse resultado é consistente com o que tem sido salientado por Whipple e Frankel (2000). Os autores advogam que, em projetos de DNS, a confiança é crucial para que o relacionamento seja um sucesso, uma vez que uma empresa depende da outra para realizar suas próprias atividades e objetivos da melhor forma.

Criar confiança é mais relevante na relação entre empresas parceiras do que entre departamentos/setores de uma mesma organização, uma vez que gestores das empresas parceiras são independentes e a empresa desenvolvedora não tem o controle direto das atividades e dos processos por elas desenvolvidos. Nessa mesma linha e uma vez que uma rede é composta por empresas de diferentes setores que trabalham ao mesmo tempo, a imagem de uma empresa no mercado torna-se uma característica relevante e que influencia a relação em rede.

Esta característica - melhoramento da imagem da empresa - está relacionada com a influência que a marca de uma empresa tem nas suas parceiras, quando elas estão atuando no mercado através da relação rede. Por exemplo, se uma pequena empresa estabelece uma parceria com uma empresa de grande dimensão, que já é conhecida no mercado e tem forte credibilidade perante os clientes, é muito provável que a empresa de menor dimensão torne-se conhecida também e que os clientes associem a imagem da empresa de menor dimensão a da empresa de grande dimensão. Porém, o oposto também pode acontecer. Caso uma empresa com uma imagem ruim no mercado estabelece parcerias, as empresas parceiras acabam por se associar à imagem negativa da parceira.

Essa característica torna-se relevante em projetos complexos de DNS, porque envolve um maior número de parcerias e a probabilidade de estabelecer parceria com empresas bem conhecidas, melhorando a imagem de um novo serviço, é maior do que em um projeto de menor dimensão.

Outra característica identificada no estudo foi a gestão da informação e comunicação. Uma comunicação forte e estruturada foi enfatizada pelos entrevistados como essencial para um relacionamento bem-sucedido no âmbito da rede colaborativa. A comunicação estruturada entre todas as empresas envolvidas no DNS permite que elas trabalhem na mesma direção, concentrando esforços para alcançar os mesmos 
objetivos. A comunicação é, também, a base para criar confiança e desenvolver uma visão compartilhada sobre o conceito de serviço. Nesse contexto, o gestor da rede deve estar apto a utilizar e gerir as informações e regularmente disseminar a informação para todas as empresas envolvidas na rede, a fim de gerar valor visando ao sucesso do relacionamento (YOSHINO, 1996; ZANINELLI; PATRÍCIO; FALCÃO e CUNHA, 2010).

Visão compartilhada significa que todos os parceiros envolvidos no processo de desenvolvimento do novo serviço devem compartilhar uma visão comum do serviço que está sendo desenvolvido. Uma visão compartilhada permite que ambos, a rede colaborativa e o projeto de um novo serviço, ocorram em harmonia, através da definição de um conjunto comum de metas alcançáveis. Nesse contexto, para que o processo de DNS ocorra da melhor forma e o serviço final seja um sucesso, os seus parceiros devem compartilhar a mesma visão sobre o conceito de serviço que estão a desenvolver. Caso não exista semelhança total em partilhar este ponto de vista, deve haver esforços para assegurar que haja, pelo menos, visão complementar, assegurando a qualidade do serviço final.

Em resumo, o estudo mostrou que alguns fatores de qualidade cruzam todo o processo do DNS, entretanto, a relevância desses fatores evolui ao longo de diferentes fases. Outros fatores são específicos de uma única fase. Esses resultados contribuem para a integração das pesquisas em torno das redes, bem como novos serviços para lidar com os novos desafios de projetos complexos de DNS. Este estudo também traz novas perspectivas sobre a forma como a gestão informação e da comunicação deve evoluir através das diferentes fases do processo de DNS, a fim de permitir o seu sucesso.

A Gestão da informação e da comunicação consiste no conjunto de atividades coordenadas que visa a: (1) obter um diagnóstico das necessidades organizacionais; (2) mapear os fluxos formais de informação nos vários setores da organização; (3) prospectar, coletar, filtrar, monitorar e disseminar informações de diferentes naturezas; (4) elaborar produtos e serviços informacionais (VALENTIN, 2005); e (5) Associar de maneira sinérgica: tecnologia e informação e comunicação (GUIMARÃES; SAMPAIO, 2002).

Dessa forma, no âmbito do processo de DNS, a gestão da informação e da comunicação busca explorar os recursos informacionais internos e externos às empresas, para que as mesmas sejam capazes de se adaptar rapidamente às mudanças do ambiente externo e interno e, o mesmo tempo, tenham habilidade de comunicar aos colaboradores tais mudanças para que esses sejam capazes de desenvolver e/ou melhorar seus produtos e serviços, com o objetivo de potencializar o processo de DNS.

As empresas que atuam no mercado e desenvolvem seus produtos e serviços através de redes colaborativas, ou seja, estabelecem parcerias com empresas externas, com o objetivo de otimizar o desenvolvimento desses, devem ter uma maior atenção quando da gestão da informação e 
da comunicação, uma vez que a complexidade do processo e os stakeholders envolvidos excedem a gestão dos processos internos (ZANINELLI, 2011). Portanto, para que seja assegurado o valor da gestão de ambos - informação e comunicação - é necessário um conjunto de tarefas bem estruturado, que esteja sob responsabilidade de um único gestor ou de uma equipe de gestão dedicada ao desenvolvimento de um único projeto DNS, devido à complexidade das atividades e tarefas envolvidas nesse processo.

\section{Conclusão}

Os resultados revelaram que a empresa desenvolvedora - dona do novo serviço - desenvolve as atividades de seu core business, ao estabelecer parcerias para as atividades consideradas secundárias. A relação de colaboração através das redes, desde as fases iniciais do processo, proporciona uma redução no seu tempo e custo e potencializa o processo, uma vez que algumas atividades são desenvolvidas por empresas especialistas.

Destarte, os resultados mostram que as redes colaborativas desempenham um papel importante em complexos projetos de novos serviços, uma vez que elas são responsáveis pela execução de várias atividades importantes, ao longo das diferentes fases do DNS. No entanto, a simples criação de uma rede não é suficiente, sua qualidade determina o impacto positivo ou negativo que isso terá sobre o desempenho do processo de DNS, portanto, os fatores de qualidade das empresas parceiras e do relacionamento entre eles indicam se as empresas possuem os atributos certos para desenvolver-se como um grupo e desempenhar com eficácia as atividades envolvidas no processo de DNS. Fatores de qualidade das redes que impulsionam o desempenho de DNS são classificados em duas dimensões: (1) características das parceiras e (2) características do relacionamento.

Os resultados do estudo mostram, também, que todos os fatores que representam a qualidade da rede são fundamentais para o bom desempenho do processo, entretanto, a ênfase foi direcionada às atividades relacionadas à gestão da informação e da comunicação - fator este indicado na dimensão 2 da Qualidade da Rede Colaborativa característica do relacionamento. Embora estatisticamente este fator não tenha sido mencionado em primeiro lugar, o foco, neste estudo, não é o número de vezes que o fator é citado $e$, sim, a intensidade que os entrevistados davam a cada fator que representa a qualidade da rede.

Por se tratar de um estudo qualitativo, o número de entrevistas (neste caso, 39) não pode ser considerado uma amostra consistente em termos numéricos, porém, com base no método adotado - Estudo de caso e Grounded Theory - respectivamente para o estudo exploratório e estudo qualitativo, a qualidade e a interpretação dos dados tornam-se mais relevantes do que o número de entrevistas. Portanto, a enfâse dada no 
aspecto gestão da informação e comunicação pode ser justificada com base na interpretação das entrevistas.

Nesse contexto e com base nos resultados obtidos no estudo em tela, apesar das características pessoais de cada empresa serem fundamentais para o fornecimento de serviços e produtos com qualidade, tais qualidades tornam-se secundárias se não existir uma boa relação entre elas e a base para atingir o sucesso da relação é uma clara comunicação e uma política de gestão da informação que seja transversal, rápida e atual entre todas as empresas membros da rede. A responsabilidade para que haja uma boa comunicação e uma intensa troca de informações entre os membros da rede é da empresa desenvolvedora, por ser ela a reponsável por contratar e gerir as diferentes redes formadas ao longo do processo de DNS.

Com base no exposto, esta pesquisa colaborou para compreender as fases, as atividades e os atores envolvidos no processo de DNS, bem como entender como e quando as redes de colaboração são formadas ao longo das diferentes fases do processo. O estudo também contribuiu para identificar os principais fatores de qualidade das redes que exercem maior influência na performance do processo de DNS e, por consequência, no serviço final. Trouxe, também, uma importante contribuição para entendermos o papel estratégico que a comunicação e a gestão da informação têm em projetos complexos que acontecem no contexto da rede. Destaca-se, também, que, apesar do estabelecimento de redes colaborativas ser uma prática cada vez mais adotada na moderna gestão de empresas, o estudo dessas práticas no contexto do DNS, até então, era encontrado na literatura de forma separada.

O próximo passo para a continuidade desta pesquisa envolve o estudo quantitativo com todos os colaboradores das empresas envolvidas na pesquisa que atuam diretamente no processo de desenvolvimento de novos serviços, com base na análise do survey questionário, para validar a importância dos fatores identificados na fase qualitativa.

\section{Limitações e sugestões para futuras pesquisas}

Estudar quatro projetos complexos de DNS que estavam em diferentes fases de desenvolvimento foi um campo de pesquisa muito rico e completo. Todos os quatro projetos em estudo têm características e dimensões semelhantes e foram desenvolvidos por uma empresa de prestígio, Portuguesa, que atua nos mercados nacionais e internacionais e, portanto, os projetos foram desenvolvidos em diferentes realidades. As características mencionadas fazem os resultados do estudo relevantes em comparação com outros da área, que investigam um único projeto no mesmo contexto.

Para futuras pesquisas, isso poderia ser expandido, não só para outros setores, mas, também, para outros tipos de projetos. Isto porque 
novas pesquisas sobre fatores de desempenho do DNS e os fatores de qualidade das redes, se estudados em novos cenários, além de analisados em novos contextos, podem ser confirmados, aplicados e adaptados a novas realidades, o que poderia envolver não apenas projetos de serviço, mas, também, de produtos.

Portanto, para outras pesquisas, sugere-se envolver não apenas as empresas que fazem parte do processo de DNS, mas, também, os consumidores finais, pois esses são parte do processo e o sucesso do serviço final depende da interação e da experiência deles em toda a oferta e consumo do serviço.

\section{Referências}

AKAMAVI, R. A research agenda for investigation of product innovation in the financial services sector. Journal of Services Marketing, v. 19, n. 6, p. 359-378, 2005.

ALAM, I.; PERRY, C. A customer-oriented new service development process. Journal of Services Marketing, v. 16, n. 6, p. 515-534, 2002.

ALBRECHT, K. Revolução nos serviços: como as empresas podem revolucionar a maneira de tratar os seus clientes. São Paulo: Pioneira, 2000.

ANAND, B. N.; KHANNA, T. Do firms learn to create value? The case of alliances. Strategic alliances: theory and evidence. Strategic Management Journal, v. 21, p. 295-316, 2004.

AUSTIN, J. E. The collaboration challenge: how nonprofits and businesses succeed through strategic alliances. San Francisco : Jossey-Bass Publishers, 2000.

CAMARINHA-MATOS, L. M.; AFSARMANESH, H. (Eds.). Collaborative Networks: reference modeling. New York: Springer, 2008.

CHARMAZ, K. Constructing grounded theory: a practical guide through qualitative analysis. Sage Publications: Thousand Oaks, 2006.

EDGETT, S. The traits of successful new service development. Journal of Services Marketing, v. 8, n. 3, p. 40, 1994.

EDVARDSSON, B. et al. New service development and innovation in the new economy. Lund: Studentlitteratur, 2000.

EDVARDSSON, B. et al. Analysis, planning, improvisation and control in the development of new services. International Journal of Service Industry Management, v. 6, n. 2, p. 24-35, 1995.

ELMUTI, D.; KATHAWALA, Y. An overview of strategic alliances. Management decision, v. 39, n. 3, p. 205-218, 2001.

FROEHLE, C. M. et al. Antecedents of new service development effectiveness. Journal of Service Research, v. 3, n. 1, p. 3, 2000. 
FROEHLE, C. M.; A. V. ROTH, A. V. A Resource process framework of new service development. Production and Operations Management, v. 16, n. 2, p. 169-188, 2007.

GRONROOS, C. Creating a relationship dialogue: communication, interaction and value. The Marketing Review, v. 1, n. 1, p. 5-14, 2000.

GUIMARÃES, M. C. S.; SAMPAIO, R. M. Desenho de um projeto para gestão da informação: um estudo exploratório na agência nacional de petróleo (ANP). In: WORKSHOP BRASILEIRO DE INTELIGÊNCIA COMPETITIVA E GESTÃO DO CONHECIMENTO,3., 2002, São Paulo. Anais... São Paulo, 2002. p. 26505.

JAW, C. et al. The determinants of new service development: service characteristics, market orientation, and actualizing innovation effort. Technovation, v. 30, n. 4, p. 265-277, 2010.

JOHNE, A.; STOREY, C. New service development: a review of the literature and annotated bibliography. European Journal of Marketing, v. 32, n. 3/4, p.184-251, 1998.

JONG, J.; VERMEULEN, P. Organizing successful new service development: a literature review. Management Decision, v. 41, n. 9, p. 844-858, 2003.

JOHNSON, S. et al. A critical evaluation of the new service development process: integrating service innovation and service design. In: FITZSIMMONS, J.; FITZSIMMONS, M. (Eds.). New Service Development: creating memorable experiences. Sage Publications: Thousand Oaks, 2000. p. 1-32.

JUNIOR, A. B. S.; RIBEIRO, Á. H. P. Parcerias e alianças estratégicas. In: BARROS, B. T. (Org.). Fusões, aquisições \& parcerias. São Paulo: Atlas; Fundação Dom Cabral, 2001. p. 89-101.

LÉON, M. E. O.; AMATO NETO, J. Redes de cooperação produtiva: uma estratégia de competitividade e sobrevivência para as pequenas e médias empresas. Gestão e Produção, v. 8, n. 3, p. 289-303, 2001.

LEWIS, J. D. Alianças estratégicas: estruturando e administrando parcerias para o aumento da lucratividade. São Paulo: Pioneira, 1992.

LIPNACK, J. ; STAMPS, J. Rede de informações. São Paulo: Makron Books, 1994.

MANSURY, M.; J. LOVE, J. Innovation, productivity and growth in US business services: a firm-level analysis. Technovation, v. 28, n. 1-2, p. 52-62, 2008.

MARTIN JR, C.; HORNE, D. Level of success inputs for service innovations in the same firm. International Journal of Service Industry Management, v. 6, n. 4, p. 40-56, 1995.

NORMANN, R. Administração de serviços. São Paulo: Atlas, 1993.

OJANEN, V. et al. New Service Development: success factors from the Viewpoint of Fleet Asset Management of Industrial Service Providers. In: 
INTERNATIONAL WORKING SEMINAR OF PRODUCTION ECONOMICS, $15 .$, March 3-7 2008. Pre-prints of the... Innsbruck, Austria, 2008. p. 369-380.

OSTROM, A. et al. Moving forward and making a difference: research priorities for the science of service. Journal of Service Research, v. 13, n. 1 , p. 4, 2010.

ROSENFELD, S. A. Bringing Business Clusters into the mainstream of economic development. Europian planning Studies, v. 5, n. 1, p. 3-23, 1997.

SAMPIERI, R. et al. Metodologia de pesquisa. São Paulo: McGraw-Hill, 2006.

SCHEUING, E.; JOHNSON, E. A proposed model for new service development. Journal of Services Marketing, v. 3, n. 2, p. 25-34, 1993.

STEVENS, E.; DIMITRIADIS, S. Managing the new service development process: towards a systemic model. European Journal of Marketing, v. 39, n. 1/2, p. 175-198, 2005.

TAX, S.; STUART, I. Designing and implementing new services: the challenges of integrating service systems. Journal of Retailing, v. 73, n. 1 , p. 105-134, 1997.

VALENTIM, M. L. P. Métodos qualitativos de pesquisa em Ciência da Informação. São Paulo: Polis, 2005.

VALKEAPAA, E. et al. New service development process in a strategic alliance. In: INTERNATIONAL PRODUCT DEVELOPMENT MANAGEMENT CONFERENCE, 13., Milano, 2006. p. 1487-1501.

VARGO, S. L.; LUSCH, R. F. Evolving to a new dominant logic for marketing. Journal of Marketing, v. 68, p. 1-17, 2004.

WHIPPLE, J. M.; FRANKEL, R. Strategic alliance success factors. Journal of Supply Chain Management, v. 36, n. 3, p. 21-28, 2000.

YIN, R. K. Case study research: design and methods. Thousand Oaks: Sage Publications, 2009.

YOSHINO, M. Y. Strategic alliances: an entrepreneurial approach to globalization. Harvard Business School Press, 1996.

ZANINELLI, T. B. New service development (NSD) process in the collaborative networks context: a study of NSD performance factors across its different stages. 2011. 277 f. Tese (Doutorado em Engenharia Industrial e Gestão) - Faculdade de Engenharia.Universidade do Porto, 2011.

ZANINELLI, T.; PATRÍCIO, L.; FALCÃO e CUNHA, J. New service Development in the Context of Collaborative Networks: a study of the influence of partnerships across the different stages of NSD process in large shopping mall development. In: INTERNATIONAL SERVICE RESEARCH CONFERENCE, june 17-19, 2010. Proceeding-AMA Servsig... Porto: School of Engineering of the University of Porto, 2010, p. 2010. 